\title{
Pour la bonne pratique du débat bioéthique
}

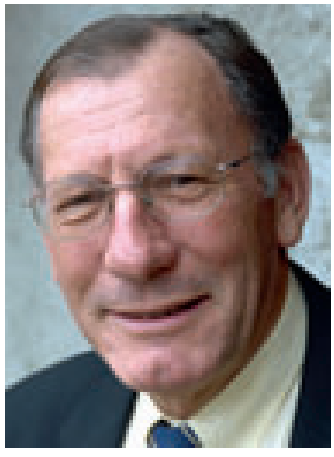

Jean Martin
En novembre dernier, j'ai participé à une réunion du Comité international de bioéthique (CIB) de l'UNESCO, suivie d'une Conférence conjointe soutenue par l'Union européenne, qui ont permis des échanges sur les expériences de comités de bioéthique dans différentes parties du monde. Le professeur d'éthique médicale J.J.M. van Delden, d'Utrecht, a fait un exposé incisif sur les conditions d'un travail fructueux dans des instances évaluant des situations médicales complexes. «Faire de l'éthique», dit-il, c'est mener une réflexion interpersonnelle systématique afin de définir la conduite responsable à adopter dans le cas soumis; avec l'objectif d'arriver à une position/ un avis dont chaque membre peut admettre le bienfondé. Pour cela, il importe d'observer du recul, une certaine humilité et du bon sens - référence à la phronésis (sagesse pratique) des Grecs. Cela m'a rappelé la formule de l'éthicien genevois Eric Fuchs selon qui l'éthique, c'est rechercher «Comment faire pour bien faire».

Les principes suivants sont particulièrement importants: débattre sereinement, s'intéresser aux arguments qui diffèrent de sa propre opinion; éviter les formulations de type directif/autoritaire et cas échéant leur résister; considérer toutes les perspectives pertinentes. Dans une commission d'éthique, on doit postuler qu'on est entre personnes raisonnables, dit van Delden; chacun a bien sûr une position de départ, à laquelle il croit, mais celle-ci reste provisoire, ne saurait être immuable (contrairement à ce qu'on voit en matière de morale, voire dans le débat politique!). Etre prêt à abandonner sa position quand la discussion montre qu'elle n'est pas appropriée dans la situation étudiée est une condition d'un travail fructueux.

Il y a là des défis pour les personnes impliquées, professionnels de santé, éthiciens, juristes, autres. En effet, la limite entre chercher à présenter de manière convaincante - mais sans myopie - une position qu'on juge bonne, d'une part, et d'autre part s'obstiner à affirmer, par exemple, que sa propre opinion est la seule rationnelle, cette limite n'est pas toujours évidente. Un de nos maîtres de santé publique disait que trois conditions de base, pour arriver à des relations de qualité, sont «Ecouter, écouter, et écouter». Et il importe de bien tenir compte du caractère pluraliste de nos sociétés.

A propos de la contribution dans ces commissions de membres affirmant des convictions religieuses ou morales fortement ancrées, l'éthicien hollandais s'est montré réservé et à vrai dire passablement critique. Il parle de «life stances» (positions de vie). Dans son expérience, si les «stances» de ces membres ne sont en aucune manière négociables (s'agissant du débat en groupe, dont on rappelle qu'il concerne d'autres personnes qu'eux-mêmes), quand leurs convictions déterminent a priori de manière définitive leur position sur un enjeu, la réflexion éthique n'est plus possible (that's where ethics ends), le travail est bloqué. Cela semble bien catégorique ... Pourtant, le fait est que, discutant un problème/dilemme biomédical, la mission de la commission n'est pas de présenter au patient et à l'équipe soignante des positions figées, mais bien de procéder à une évaluation large de la situation, tenant compte des différents intérêts et valeurs en cause, et de suggérer les pistes possibles en vue des décisions à prendre.

Pour Fernando Lolas, du Bureau américain de l'OMS, il est nécessaire en bioéthique d'aller au-delà de positions philosophiques monologiques pour tendre vers une démarche «polyphonique» - passant cas échéant de l'émotion à une proposition réfléchie et pondérée. Un autre orateur latino-américain a insisté sur le fait que le débat devait être critique et séculier.

A première vue, les défis bioéthiques semblent surtout liés aux avancées scientifiques et technologiques dans les pays industrialisés et moins dans les régions sous-médicalisées. Rien n'est moins vrai, a dit le professeur Evariste Likinda, un neurochirurgien qui préside le Comité de bioéthique de la République démocratique du Congo. Développements et problèmes sont chaque jour plus mondialisés. Penser par exemple aux études (trials) médico-pharmaceutiques entrepris à large échelle dans les pays pauvres - le plus souvent pour tester des produits destinés aux populations solvables du Nord; au Brésil par exemple, 250000 personnes sont incluses dans de telles études. Les questions soulevées, entre autres les protections que doivent garantir les protocoles, ont été évoquées avec force; garder à l'esprit que les sujets de recherche sont souvent particulièrement vulnérables, y compris du point de vue de leur capacité à comprendre toutes les informations relatives à leur participation.

Jean Martin* du Comite internation et membre de la rédaction du BMS. 